\section{Sensitivity of Si-based zero-bias backward diodes for microwave detection}

S.-Y. Park, R. Yu, S.-Y. Chung, P.R. Berger, P.E. Thompson and P. Fay

\begin{abstract}
Silicon-based backward diodes incorporating $\delta$-doped active regions for direct detection of microwave radiation with zero external DC bias have been demonstrated at room temperature and characterised for their sensitivity. The resulting backward diodes, which were grown by low temperature molecular beam epitaxy, show a high zero-bias curvature coefficient $(\gamma)$ of $23.2 \mathrm{~V}^{-1}$ with a junction resistance $\left(R_{j}\right)$ of $687 \mathrm{k} \Omega$ for a $5 \mu \mathrm{m}$ diameter mesa diode. The microwave-frequency voltage sensitivity is reported for the first time; a measured sensitivity of $2376 \mathrm{~V} / \mathrm{W}$ is obtained at zero-bias when driven from a $50 \Omega$ source. An intrinsic $3 \mathrm{~dB}$ cutoff frequency of $1.8 \mathrm{GHz}(5 \mu \mathrm{m}$ diameter) was determined based on an extracted series resistance of $290 \Omega$ and a junction capacitance of $0.307 \mathrm{pF}$ using a small-signal model established to fit the measured $S$-parameters.
\end{abstract}

Introduction: Si-based backward diodes are a leading candidate for passive detection of millimetre-wave radiation. Their high zero-bias nonlinearity enables operation with no extra bias control circuits required, which dramatically reduces $1 / f$ noise. Monolithic integration and registration with complementary metal-oxide-semiconductor (CMOS) or SiGe heterojunction bipolar transistor (HBT) readout circuitry is poised to leverage the silicon semiconductor industry for potential widespread usage of these sensor elements for security applications such as detection of concealed weapons.

Backward diodes, based on tunnelling and proposed by Esaki and Miyahara in the early 1960s [1], have been realised epitaxially, and are very good candidates for microwave detection compared to several alternative detector technologies in terms of their high sensitivity, low noise, simplicity and room temperature operation. A tunnelling backward detector enables the direct detection of microwave radiation without external applied bias, therefore no extra bias control circuits are required, resulting in a simplified system and reduced pixel complexity. This leads to a significant reduction of the total cost of fabrication and assembly [2]. Most backward-diode detectors utilise III-V compound-based heterojunctions, which are not readily compatible with mainstream silicon technology. Recently, the first high sensitivity Si-based backward diodes, grown by low-temperature molecular beam epitaxial (LT-MBE) growth, were demonstrated by Jin et al. [3], who reported a record Si-based curvature coefficient of $31 \mathrm{~V}^{-1}$ at zero bias, which is almost double that of a commercial discrete $\mathrm{Ge}$ backward diode at room temperature [4].

In this Letter, we report the first directly-measured microwave sensitivity performance of a zero-bias Si-based backward detector. A measured sensitivity of $2376 \mathrm{~V} / \mathrm{W}$ was determined when driven from a $50 \Omega$ source. A cutoff frequency of $1.8 \mathrm{GHz}$ was extracted with a series resistance of $290 \Omega$ and a junction capacitance of $0.307 \mathrm{pF}$ for a $5 \mu \mathrm{m}$ diameter mesa device. Here the structure was modified from the previous report [3] to reduce the junction resistance for better RF performance without a significant reduction in the zero-bias curvature coefficient.

Experiment: The schematic diagram of the Si-based backward diode structure used in this study is shown in Fig. 1a. Epitaxial growth was performed by low-temperature molecular beam epitaxy (LT-MBE) using elemental $\mathrm{Si}$ and $\mathrm{Ge}$ in electron-beam sources on $\mathrm{Si}(100)$ substrates $(<0.005 \Omega \mathrm{cm})$. $p$ - and $n$-type doping were achieved by evaporation from $\mathrm{B}$ and $\mathrm{GaP}$ in Knudsen cells, respectively. The backward diode structure utilised a Si buffer layer grown at $650^{\circ} \mathrm{C}$ on the $\mathrm{Si}$ substrate. The $p^{+}-\mathrm{Si}$ substrate contact layer $\left(5 \times 10^{19} \mathrm{~cm}^{-3}\right)$ was grown at $500^{\circ} \mathrm{C}$. A $1 \mathrm{~nm} \mathrm{SiGe} \mathrm{cladding} \mathrm{layer} \mathrm{is} \mathrm{inserted} \mathrm{below} \mathrm{the}$ contact layer to suppress B outdiffusion, followed by a B $\delta$-doping layer $\left(2.5 \times 10^{13} \mathrm{~cm}^{-2}\right)$ deposited while the growth temperature was lowered from 500 to $320^{\circ} \mathrm{C}$. Next, the active tunnelling region, a $4 \mathrm{~nm}$ undoped $\mathrm{Si}_{0.6} \mathrm{Ge}_{0.4}$ spacer layer and a $2 \mathrm{~nm}$ undoped $\mathrm{Si}$ layer, was grown at $320^{\circ} \mathrm{C}$, followed by the deposition of a $\mathrm{P} \delta$-doping layer $\left(2.5 \times 10^{13} \mathrm{~cm}^{-2}\right)$. The top $n^{+}$contact layer was grown at $400^{\circ} \mathrm{C}$ and consisted of a $100 \mathrm{~nm} n^{+}$-Si layer $\left(5 \times 10^{19} \mathrm{~cm}^{-3}\right)$, a P $\delta$-doping layer $\left(1 \times 10^{14} \mathrm{~cm}^{-2}\right)$, and a $5 \mathrm{~nm}$ undoped Si layer [5].

Prior to device fabrication, portions of the MBE wafer were annealed, separately, in a rapid thermal annealing (RTA) furnace at various temperatures for $60 \mathrm{~s}$ under a forming gas ambient $\left(\mathrm{N}_{2} / \mathrm{H}_{2}\right)$. The anneal removes point defects, induced by the LT-MBE process, which significantly degrade the curvature coefficient [3]. Annealing temperatures between 850 and $890^{\circ} \mathrm{C}$ were investigated to determine the optimum annealing condition for a low junction resistance concurrently with a high curvature coefficient at zero bias.

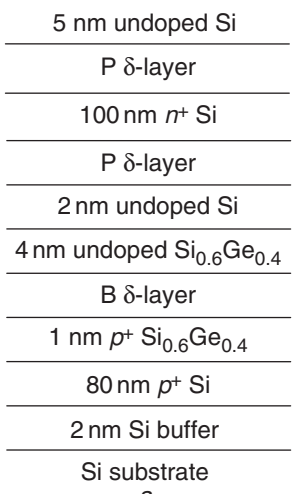

a

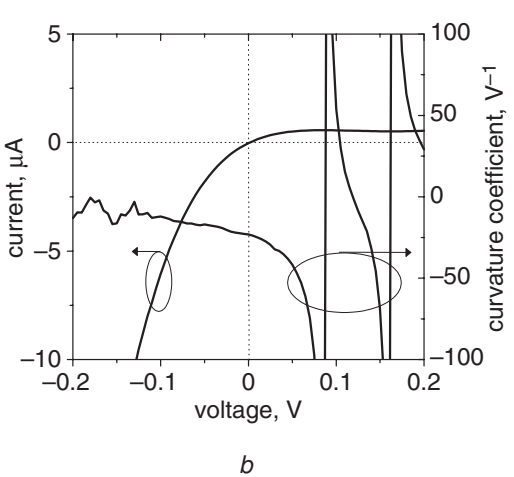

Fig. 1 Schematic diagram of Si/SiGe backward diode structure grown by LT-MBE, and room temperature I-V characteristic and curvature coefficient $(\gamma)$ against DC bias voltage for $5 \mu \mathrm{m}$ diameter $\mathrm{Si} / \mathrm{SiGe}$ backward diode

a Schematic diagram

$b$ Room temperature $I-V$ characteristic and curvature coefficient $(\gamma)$ against DC bias voltage

Device fabrication included circular cathode contact definition followed by deposition and lift-off of $\mathrm{Ti} / \mathrm{Cr} / \mathrm{Au}(150 \AA / 1500 \AA$ / $1000 \AA$ ) metal using electron beam evaporation. Self-aligned mesas were formed using wet etching in a $\mathrm{HF} / \mathrm{H}_{2} \mathrm{O} / \mathrm{HNO}_{3}(1: 100: 100)$ solution. The anode ohmic contact metal of Pt/Au $(150 \AA / 1000 \AA)$ was defined by lift-off photolithography and subsequent electron beam evaporation. A photo-definable polyimide process was then used to form a dielectric isolation layer to fashion via holes. Finally, the last lithography step defined the ground-signal-ground probe pads needed for RF measurement by deposition and lift-off of Ti/Au (150 ̊/2000 $)$.

Results: Fig. $1 b$ shows the room temperature $I-V$ characteristic of a $5 \mu \mathrm{m}$ diameter $\mathrm{Si} / \mathrm{SiGe}$ backward diode illustrating a strong nonlinearity with a curvature coefficient $\left(\gamma=\left(d^{2} I / d^{2} V\right) /(d I / d V)\right)$ of $23.2 \mathrm{~V}^{-1}$, and an areal junction resistance of $13.5 \mathrm{M} \Omega \mu \mathrm{m}^{2}$ at zero bias. This compares favourably with the previous report [3] of a curvature coefficient of $31 \mathrm{~V}^{-1}$ and an areal junction resistance of $140 \mathrm{M} \Omega \mu \mathrm{m}^{2}$. In the previous report [3], $18 \mu \mathrm{m}$ diameter $\mathrm{Si} / \mathrm{SiGe}$ backward diodes grown at 320 and $400^{\circ} \mathrm{C}$ exhibited a junction resistance, $R_{j}$, of 22 and $550 \mathrm{k} \Omega$, respectively. This is contrasted to a junction resistance of only $4 \mathrm{k} \Omega$ for the devices reported here of the same dimensions. The RF sensitivity $\left(\beta_{V}\right)$ is directly proportional to the DC nonlinearity curvature coefficient through the approximation $\beta_{V}=2 Z_{s} \gamma$, where $Z_{s}$ is the source impedance.

$\mathrm{Si} / \mathrm{SiGe}$ backward diode detectors were characterised using on-wafer $S$-parameter measurements from 0.01 to $20 \mathrm{GHz}$ in $0.34 \mathrm{GHz}$ steps. De-embedding of the device from pad parasitics was based on $S$ parameter measurement of on-wafer short- and open-pad test structures. The measured data was fitted to a small-signal equivalent circuit model using Advanced Design System (ADS) software so as to obtain the intrinsic device parameters including series resistance $\left(R_{S}\right)$, junction capacitance $\left(C_{j}\right)$ and junction resistance $\left(R_{j}\right)$. The intrinsic cutoff frequency, $f_{c}=1 /\left(2 \pi R_{s} C_{j}\right)$, at zero bias for a $5 \mu \mathrm{m}$ diameter mesa diode is estimated to be $1.8 \mathrm{GHz}$ based on an extracted series resistance of $290 \Omega$ and junction capacitance of $0.307 \mathrm{pF}$. This is limited in these first-generation devices by the ohmic contact resistances and by capacitive coupling to the conductive substrate.

Voltage sensitivity of these $\mathrm{Si} / \mathrm{SiGe}$ backward diodes, defined as the DC voltage output developed across the detector divided by the available RF power incident on the detector, was measured on-wafer from 0.01 to $20 \mathrm{GHz}$ against input power level. Fig. 2 shows the measured sensitivity and detector voltage characteristics of $5 \mu \mathrm{m}$ mesa diameter diodes against input power at an RF frequency of $1 \mathrm{GHz}$. A $1 \mathrm{~dB}$ compression point of $-10.26 \mathrm{dBm}$ is observed. Table 1 shows the 
measured sensitivity against frequency from 0.01 to $20 \mathrm{GHz}$ for a $5 \mu \mathrm{m}$ diameter mesa detector driven by a $50 \Omega$ source. The data in Table 1 indicate the device is well-modelled by a one-pole frequency response with an extrinsic $3 \mathrm{~dB}$ frequency of $1.2 \mathrm{GHz}$. This is consistent with the small-signal model obtained from measured $S$-parameters.

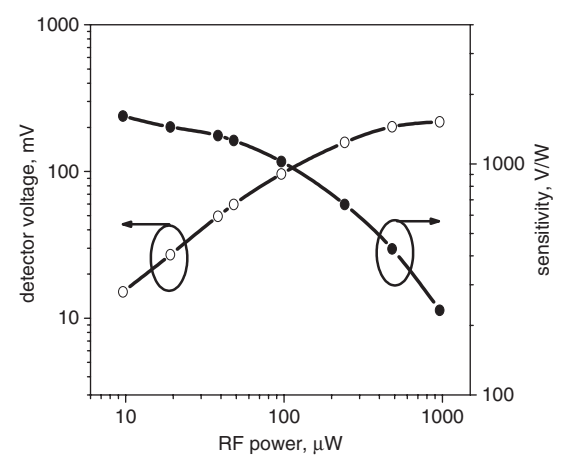

Fig. 2 Measured sensitivity and detector voltage characteristics of $5 \mu \mathrm{m}$ diameter mesa against input power at RF frequency of $1 \mathrm{GHz}$.

Table 1: Sensitivity measurement against frequency from 0.01 to $20 \mathrm{GHz}$ for $5 \mu \mathrm{m}$ diameter mesa detector driven by $50 \Omega$ source

\begin{tabular}{|c|c|}
\hline Frequency $(\mathrm{GHz})$ & Sensitivity $(\mathrm{V} / \mathrm{W})$ \\
\hline 0.01 & 2376 \\
\hline 0.1 & 2344 \\
\hline 0.2 & 2113 \\
\hline 0.5 & 2022 \\
\hline 1 & 1655 \\
\hline 2 & 1386 \\
\hline 5 & 628 \\
\hline 10 & 269 \\
\hline 20 & 182 \\
\hline
\end{tabular}

Conclusion: Si-based backward diodes incorporating $\delta$-doped active regions were grown by low temperature molecular beam epitaxy (LT-MBE) as zero bias detectors with high sensitivity and strong curvature coefficient. They were fabricated and the RF sensitivity measured for the first time. The maximum measured voltage sensitivity was $2376 \mathrm{~V} / \mathrm{W}$ when driven with a $50 \Omega$ source, which results in an intrinsic cutoff frequency of $1.8 \mathrm{GHz}$ for a $5 \mu \mathrm{m}$ diameter mesa diode. The measured $I-V$ characteristics of the backward diodes show a high zero-bias curvature coefficient $(\gamma)$ of $23.2 \mathrm{~V}^{-1}$ with an areal junction resistance of $13.5 \mathrm{M} \Omega \mu \mathrm{m}^{2}$. The junction resistance is one order of magnitude smaller than a previous report [3], leading to improved prospects for impedance matching in system applications.

Si-based backward diodes as zero bias detectors should be capable of cutoff frequency within the atmospheric window of $94 \mathrm{GHz}$ for passive millimetre-wave detection with the usage of a low resistance Ni silicide ohmic technology [6], scaled size devices, and high-resistivity substrates.

Acknowledgments: The work done at The Ohio State University was supported by the Ohio State Office of Homeland Security and the National Science Foundation. The work performed at the Naval Research Laboratory was supported by the Office of Naval Research. The authors are indebted to C. L. Cerny and J. E. Van Nostrand of the Air Force Research Laboratory for discussions and support.

(C) The Institution of Engineering and Technology 2007

29 January 2007

Electronics Letters online no: 20070299

doi: 10.1049/el:20070299

S.Y. Park, S.-Y. Chung and P.R. Berger (Department of Electrical and Computer Engineering, The Ohio State University, Columbus, OH 43210-1272, USA)

E-mail: pberger@ieee.org

R. Yu (Department of Physics, The Ohio State University, Columbus, $\mathrm{OH}$ 43210-1106, USA)

P.E. Thompson (Naval Research Laboratory, Code 6812, Washington, DC 20375-5347, USA)

P. Fay (Department of Electrical Engineering, The University of Notre Dame, Notre Dame, IN 46556, USA)

P.R. Berger: Also with Department of Physics, The Ohio State University, Columbus, OH 43210-1106, USA

\section{References}

1 Esaki, L., and Miyahara, Y.: 'A new device using the tunneling process in narrow $p$-n junctions', Solid-State Electron., 1960, 1, pp. 13-14

2 Fay, P., et al.: 'High-performance antimonide-based heterostructure backward diodes for millimeter-wave detection', IEEE Electron Device Lett., 2002, 23, pp. 585-587

3 Jin, N., et al.: 'High sensitivity Si-based backward diodes for zero-biased square-law detection and the effect of post-growth annealing on performance', IEEE Electron Device Lett., 2005, 26, pp. 575-578

4 Burrus, C.A.: 'Backward diodes for low-level millimeter-wave detection', IEEE Trans. Microw. Theory Tech., 1963, 11, pp. 357-362

5 Jin, N., et al.: 'Diffusion barrier cladding in $\mathrm{Si} / \mathrm{SiGe}$ resonant interband tunneling diodes and their patterned growth on PMOS source/drain regions', IEEE Trans. Electron Devices, 2003, 50, pp. 1876-1884

6 Chung, S.Y., et al.: ' $\mathrm{Si} / \mathrm{SiGe}$ resonant interband tunnel diode with $\mathrm{f}_{\mathrm{r} 0}$ $20.2 \mathrm{GHz}$ and peak current density $218 \mathrm{kA} / \mathrm{cm}^{2}$ for K-band mixed-signal applications,', IEEE Electron Device Lett., 2006, 27, pp. 364-367 